\title{
Spububaúde
}

\section{Fraudes em alimentos industrializados}

\author{
Fraud in industrialized foods
}

\section{Sarah Ramila Batista de Oliveira ${ }^{1^{*}} \bullet$, Lucas D’ Lúcio Sousa Moraes ${ }^{1}$, Cristina Pacheco Coelho ${ }^{2} \bullet$}

${ }^{1}$ Graduandos em Biomedicina da Faculdade Única, Ipatinga, Minas Gerais, Brasil. ${ }^{2}$ Docente da Faculdade Única. Ipatinga, Minas Gerais, Brasil. *Autor para correspondência. E-mail: sarah.luizgustavo2016@outlook.com

\begin{abstract}
Resumo: Introdução: Atualmente a população mudou seus hábitos alimentares, e busca a cada dia alimentos industrializados, pela falta de tempo para se preparar uma alimentação saudável. E isso acarreta maus hábitos alimentares, e até mesmo o consumo de alimentos de qualidade duvidosa. As indústrias têm recebido grande demanda para industrialização de alimentos, por causa do excesso de consumo da população. Com isto as empresas têm visado em seus lucros, e em obter mais benefícios, e através disso tem se observado a cada dia que as empresas têm fraudado mais os alimentos. Objetivo: O trabalho teve como objetivo descrever o conceito de fraude alimentar, retratar os tipos de fraudes, detalhar a característica de cada fraude e por fim, mencionar os possíveis riscos à saúde, que estes alimentos são capazes de provocar ao consumidor. Revisão: A metodologia empregada neste presente trabalho trata-se de uma revisão bibliográfica realizada entre maio e junho de 2020. As bases de dados utilizadas foram SciELO e Google Acadêmico. Foram incluídos artigos do ano de 2010 até o ano de 2020.

Considerações finais: As fraudes ocorridas nas indústrias, se classifica em alterações tais como: enzimáticas, químicas, microbiológicas, microbianas, por adulterações que é adição, ou subtração e falsificações, isso ocorre para a obtenção de mais lucros, e para aumentar a validade dos alimentos, entre outras mais irregularidades. As fraudes alimentares trazem um risco para a saúde, e em casos mais graves pode levar o indivíduo a óbito. As fraudes omitem os valores nutricionais dos alimentos, e a real composição dos alimentos.
\end{abstract}

Palavras-chave: adulterações, alterações, falsificações, fraudes, indústrias.

\begin{abstract}
Introduction: Currently the population has changed their eating habits, and seeks every day industrialized foods, for the lack of time to prepare a healthy diet. And this entails bad eating habits, and even the consumption of foods of dubious quality. The industries have received great demand for food industrialization, because of the overconsumption of the population. With this companies have aimed at their profits, and in obtaining more benefits, and through this it has been observed every day that companies have defrauded more food. Objective: The objective of this work was to describe the concept of food fraud, to portray the types of fraud, to detail the characteristic of each fraud and finally to mention the possible health risks that these foods are capable of causing to the consumer. Review: The methodology used in this work is a bibliographic review carried out between May and June 2020. The databases used were SciELO and Google Scholar. Articles from 2010 to 2020 were included. Final considerations: The frauds that occur in the industries, is classified in changes such as: enzymatic, chemical, microbiological, microbial, by adulterations that is addition, or subtraction and counterfeits, this occurs to obtain more profits, and to increase the shelf life of food, among other irregularities. Food fraud brings a health risk, and in more severe cases it can lead to the individual dying. Fraud omits the nutritional values of food, and the actual composition of food.
\end{abstract}

Keywords: adulteration, alterations, forgeries, fraud, industries.

\section{Introdução}

A fraude alimentar apresenta uma motivação econômica, sendo capaz de causar danos à saúde pública, sendo relacionada em diversos tipos de fraudes, entre estas, estão aquelas que são realizadas por meio da diluição (por exemplo, a adição de água ao leite), da substituição (do azeite por óleo), da ocultação (uso de corantes e químicos para ocultar defeitos nos alimentos), da rotulagem indevida (validades ou origem alteradas), das práticas ilegais/roubo (produtos de mercado negro), das alterações não autorizadas (por exemplo, de aditivos não autorizados), e também da falsificação (caso de um vinho comum apresentado com marca valorizada) (Filipe, 2019).

Compreendendo o que foi dito, tudo que for relacionado com a substituição, a adição, adulteração ou falsificação de alimentos, ingredientes alimentícios ou embalagens de alimentos e ou declarações falsas ou enganosas, pode ser considerado uma fraude, que é classificada como um crime. Toda e qualquer fraude 
alimentar tem como objetivo o lucro, cujos os riscos que estes produtos podem causar para a saúde pública, são muitas vezes maiores, que a própria motivação econômica, não só prejudicando a saúde da população, mas como também afetando a empresa, na qual, realizou este ato criminoso. Pode ser levado em conta também, a perda de confiança por parte dos investidores, clientes, consumidores e autoridades do referente produto fraudado (Figueira, 2018).

O crime alimentar é caracterizado sempre que a escala e o impacto potencial da atividade são impactantes e cuja atividade criminosa tem um alcance regional, nacional ou internacional, o que se torna, um risco significativo para a segurança pública, ou perda financeira substancial para consumidores, ou empresas. A fraude alimentar é mais comum em alimentos líquidos do que nos sólidos, ao se tratar de alimentos com vários ingredientes comparativamente aos mais simples, de ingrediente único. As oportunidades de fraude alimentar e adulteração são grandes, devido à grande quantidade de alimentos produzidos, exportados e importados, e ao alto valor agregado de produtos específicos (Correia, 2018).

De acordo com alguns estudos, as regiões geográficas com alta vulnerabilidade à fraude alimentar, estão presentes em áreas com instabilidade política, social e uma população grande e crescente. Neste caso, possivelmente o Brasil atende a muitas dessas condições e portanto, é propenso a casos de fraudes alimentares e adulterações (Tibola et al., 2018).

Portanto, este trabalho tem como objetivo, descrever o conceito de fraude alimentar, retratar os tipos de fraudes, detalhar a característica de cada fraude e por fim, mencionar os possíveis riscos à saúde, que estes alimentos são capazes de provocar ao consumidor.

\section{Revisão e discussão}

Trata-se de um estudo de natureza descritiva, quantitativo utilizando como método a revisão bibliográfica, realizada entre maio e junho de 2020. O presente artigo é uma revisão bibliográfica que teve como objetivo buscar artigos que exemplificassem e descrevessem as fraudes que são causados em alimentos, a sua definição e os principais tipos de fraudes. O período de coleta dos artigos produzidos, foi do ano 2010 até 2020 nas seguintes bases de dados: SciELO e Google Acadêmico. Para a seleção dos estudos, restringiu-se aos realizados no Brasil e nos Estados Unidos, a análise iniciou-se com a leitura dos títulos dos estudos. No segundo momento, foram lidos os resumos e selecionados como critérios de inclusão, artigos científicos originais, aqueles que tinham como palavras-chave fraudes, indústrias, alterações, adulterações e falsificações. Por fim, foi realizada a leitura dos textos selecionados pelos critérios de inclusão e analisados de acordo com a proposta do estudo.

\section{Definição de fraude alimentar}

A fraude alimentar é uma preocupação por parte das autoridades responsáveis pelo controle de alimentos e também para os consumidores, pois os mesmos são expostos diariamente ao risco de contaminação por um produto violado ou adulterado. Estes alimentos podem causar uma intoxicação ou um agravo a saúde do consumidor. Portanto, são necessárias medidas de inspeções pelos órgãos públicos, para detectar qualquer fraude desta espécie. Esses órgãos devem penalizar os autores deste crime e fiscalizar estes produtos. Dessa forma, a garantia de uma proteção para os consumidores é assegurada (Filipe, 2019).

De uma forma geral, fraude alimentar refere-se à substituição ou adição deliberada e até intencional de uma substância ao um produto com intuito de aumentar o valor do produto e o ganho econômico, não tendo a intenção de provocar danos na saúde e nem algum tipo de prejuízo a empresa, mas quando a fraude alimentar é capaz de prejudicar a saúde do consumidor, torna-se um problema de saúde pública, afligindo a segurança alimentar e também considerada como um crime (Spink \& Moyer, 2011).

De acordo com Ribeiro (2013) tudo que se desvia das características normais, incluindo peso e preço de determinado alimento é considerado fraude. No entanto, o termo "características normais" é discutível do ponto de vista comercial e industrial. Muitos produtos com características de cor, gosto e textura diferente daquela que se deveria esperar de um produto isento de qualquer artifício técnico, já estão inclusos na alimentação humana. Dessa forma, considera-se fraude os artifícios usados sem o consentimento oficial, resultado da desnaturação de um produto, visando lucro ilícito e que não fazem parte de uma prática universalmente aceita. As fraudes alimentares podem ser feitas de diferentes formas e podem dividir-se em três grupos principais, entre eles estão: fraudes por adulteração, fraudes por falsificação, fraudes por alteração. 


\section{Fraudes por adulteração}

De acordo com Silva (2017a), nos últimos anos a adulteração dos alimentos se tornou um problema grave, pois baseia-se em atos intencionais por parte das empresas, tornando-se o alimento impuro, impróprio para o consumo, alguns alimentos são mais susceptíveis a adulteração no mercado. Temos como exemplo o café, o leite, o azeite, o mel, o suco de laranja, o suco de maçã e o açafrão. Esses são alguns exemplos de alimentos que mais sofrem adulterações em sua composição.

Esses alimentos quando são ingeridos e estão adulterados, os nutrientes necessários e reais para sua saúde humana, são afetados por essas adulterações. Contudo, podem acarretar muitos malefícios a saúde, devido à adição de um produto totalmente prejudicial à saúde. Essas adulterações ocorrem, com o intuito de baixar o custo de fabricação, o rendimento do produto, a conservação estendida, obtenção de lucros e vários outros benefícios para os produtores (Correia, 2018).

O consumidor deve ficar atento a tabela nutricional dos alimentos e aos rótulos, para assessorar no controle de qualidade nos mercados, a fim de combater essas fraudes alimentares (Martins, 2016).

\section{Fraudes por falsificação}

A falsificação de alimentos ocorre com o objetivo de aumentar o ganho financeiro das empresas. A falsificação é o ato de copiar um alimento para obter vantagens, com isso enganar o consumidor a adquirir um alimento de qualidade inferior do proposto. $\mathrm{O}$ alimento falsificado tem a aparência e as características de um produto realmente original, e legítimo (Correia, 2018).

Conforme Santos (2017) esse tipo de fraude é realizado de diferentes formas como por exemplo, quando os comerciantes que vendem carne de corte de segunda como se fosse de primeira. Outros exemplos estão no peso de algum alimento adulterado, na adição de formol e de água no leite.

Esse delito de falsificar alimentos é crime e está previsto no artigo 272 do Código penal. É um problema que preocupa as autoridades do controle alimentício, devido aos riscos que a população que consome esses alimentos é exposta (Oliveira, 2016).

\section{Fraudes por alteração}

Segundo Santos (2017) quando existem alterações em algumas ou todas as características essenciais dos alimentos, a fraude é detectada. A alteração dos alimentos só é considerada fraude, se o vendedor permitir que este alimento seja comercializado, sabendo que se encontra em condições impróprias para consumo. As alterações podem ser classificadas em alterações enzimáticas que são aquelas que originam ranço hidrolítico e escurecimento enzimático, alterações por agentes químicos, tais como, o escurecimento químico e ranço oxidativo, alterações por agentes físicos como a temperatura e luz solar, alterações microbianas pela entrada de roedores, insetos, entre outros aos alimentos e alterações por microrganismos como bactérias, fungos e leveduras.

A alteração também pode ser caracterizada sem haver interferência humana, mas pode ser causada por agentes físicos, químicos, biológicos ou enzimáticos. Essas medidas acontecem pelo simples fato das normas técnicas do produto, serem negligenciadas, ignoradas e desobedecidas, mas só se configura como fraude, se porventura o comércio souber da qualidade duvidosa do alimento e os comercializa normalmente (Figueira, 2018).

\section{Os riscos para a saúde humana}

Segundo a Legislação Brasileira, fraudar, adulterar produtos alimentícios destinados ao consumo, tornando-o ofensivo à saúde ou diminuindo o seu valor nutritivo, é considerado crime grave. Ministério da Agricultura Pecuária e Abastecimento (MAPA, 2017).

A pessoa que comete esse crime, está sujeito a pena de reclusão de quatro a oito anos e multa. As mesmas penas são aplicadas em quem fabrica, vende, expõe à venda, importa, guarda em depósito para vender, distribui ou entrega para o consumo o produto fraudado (Magalhães \& Souza, 2018).

Esses incidentes trazem consigo impactos irreparáveis, gera perdas econômicas significativas e a preocupação com a saúde humana além disso, fraudes alimentares e adulterações minam a confiança dos consumidores. Dessa forma, prejudica os estabelecimentos que comercializam o produto fraudado, acarreta imensas perdas financeiras, já que a maioria dos consumidores, sabe que o mesmo vende produtos de procedência duvidosa, podem evitar de comprar neste local (OMC, 2017).

Por esse motivo, observamos a importância de estudar e pesquisar sobre assuntos referentes a fraude alimentar, porque a mesma pode ocasionar ameaças a saúde pública. As doenças ou a intoxicação alimentar, são as consequências mais comuns dessas fraudes (Moyer et al., 2017). 
De acordo com Silva (2017b), podemos citar como exemplo de adulteração nos dias atuais, a adição do formol no leite, alterando as características físico-químicas e nutritivas do mesmo, trazendo consequências graves para saúde do indivíduo, pois o formol é tóxico se ingerido, inalado ou se tiver contato com a pele e é considerado cancerígeno pela Agência Internacional de Pesquisa do Câncer. O câncer de nasofaringe, nasossinusal e a leucemia, podem ser exemplos de doenças causadas pela ingestão de formol a longo prazo.

A alteração no mel, onde acrescenta-se água, amidos e glicoses no mesmo, a fim de render o produto e aumentar seu constituinte sólido e, consequentemente, aumentar o lucro com a venda do mel adulterado (Aguiar, 2018). A glicose, por exemplo, é o monossacarídeo responsável pela granulação do mel e, com a precipitação de açúcar, há o aumento do teor de umidade na fase líquida, o que estimula o desenvolvimento de leveduras osmofílicas, os quais são microrganismos perigosos, já que produzem metabólitos tóxicos, provocando a fermentação natural e tornando o produto improprio para o consumo (Medeiros \& Souza, 2016).

Vale a pena ressaltar a autenticidade dos alimentos, pois é de fundamental importância tanto para os consumidores quanto para as indústrias alimentícias, manter a segurança em todas as etapas de produção, desde a compra de matérias-primas até a distribuição de produtos acabados em todo o mundo (Dong et al., 2016).

As normas de autenticidade que são regularmente aplicadas através de toda a cadeia produtiva, ajudam a prevenir a ocorrência de fraudes alimentares e eventos de adulteração. No entanto, ainda estão em curso discussões a nível internacional sobre a adequação das ferramentas utilizadas para avaliar a vulnerabilidade das cadeias de fornecimento à fraude e adulteração de alimentos. Não se pode determinar quais mecanismos serão mais eficazes para o comércio global e as cadeias de abastecimento (OMC, 2017).

\section{Considerações finais}

A fraude alimentar é um tema de preocupação atualmente, pois a cada dia as indústrias tem adulterado os alimentos. A fraude ocorre, quando as empresas veem nela uma oportunidade para a obtenção de fins lucrativos. A população tem aumentado, com isso uma grande demanda de alimentos para as empresas produzirem. A maioria das fraudes ocorrem em atos intencionais por parte das empresas, sem a preocupação com a saúde pública e do seu consumidor, a fraude alimentar é um crime e está prevista no Código penal.

\section{Referências}

Aguiar, A. C. D. S. 2018. Panorama e perspectivas da cadeia produtiva do mel no Brasil. Trabalho de conclusão de curso. Uberlandia, MG: Universidade Federal de Uberlanida.

Correia, A. M. N. 2018. Fraude Alimentar: fatores de risco e medidas de controlo e prevenção. Tese de doutorado. Lisboa, PT: Universidade Nova de Lisboa.

Dong, H., Luo, D., \& Luo, H. 2016. Uso efetivo da rastreabilidade de alimentos nas cadeias de suprimento de grãos de cereais. In M. Espiñeira \& F.J. Santaclara (Eds.), Avanços nas técnicas e tecnologias de rastreabilidade de alimentos: Melhorando a qualidade em toda a cadeia alimentar. Cambridge, UK: Woodhead Publishing.

Figueira, L. C. 2018. Os conceitos de defesa dos alimentos (Food Defense) e fraude em alimentos (Food Fraud) aplicados em fábrica de temperos cárneos: um estudo de caso. Tese de doutorado. São Pulo, SP: Universidade de São Paulo.

Filipe, A. F. P. 2019. Avaliação da vulnerabilidade à fraude alimentar: o caso do Talho Nacional. Dissertação de Mestrado. Èvora, PT: Universidade de Évora).

Magalhães, L. A. L., \& Souza, S. M. O. 2018. Fraudes e adulterações no leite. Anais do 14 Simpósio de TCC e 7 Seminário de IC da Faculdade ICESP,1551-1557.

Martins, S. A. C. 2016. Avaliação da vulnerabilidade à fraude alimentar segundo o referencial BRC versão 7 . Tese de doutorado. Braga, PT: Universidade do Minho.

Medeiros, D., \& Souza, M. F. 2016. Contaminação do mel: a importância do controle de qualidade e de boas práticas apícolas. Atas de Ciências da Saúde, 3(4).

Ministério da Agricultura Pecuária e Abastecimento [MAPA]. 2017. Decreto n. 9.013, de 29 de março de 2017. Regulamenta a lei $\mathrm{n}^{\circ} 1.283$, de 18 de março de 1989 que dispõe sobre a inspeção industrial e sanitária de produtos de origem animal. Diário Oficial da União. Brasilia, DF.

Moyer, D. C, Devries, J. W, \& Spink, J. 2017. A economia de um incidente de fraude alimentar - estudos de caso e exemplos, incluindo melanina no glúten de trigo. Controle de Alimentos, 71, 358-364. 
Oliveira, M. A. M. D. 2016. Segurança na Cadeia alimentar: estudo de fraudes. Tese de doutorado. Porto, PT: Instituto Politécnico do Porto. Escola Superior de Tecnologia e Gestão.

Organização Mundial do Comércio [OMC]. 2017. Revisão estatística do comércio mundial. Recuperado de: https://www.wto.org/english/res_e/statis_e/wts2017_e/wts2017_e.pdf. Acesso em: 11 dez. 2020.

Ribeiro, S. N., \& Marcello, T. M. 2013. Avaliação da perda líquida no degelo de filés de tilápia realizada por desglaciamento. Monografia. Londrina, PR: Universidade Tecnológica Federal do Paraná.

Santos, M. M. D. D. 2017. Fraude Alimentar: análise dos resultados obtidos de amostras não conformes do género alimentício Mel. Dissertação de mestrado. Lisboa, PT: Faculdade de Medicina Veterinária.

Silva, L. R. R. 2017. Fraude no leite: experimento investigativo para o ensino de química. Fraude no leite: experimento investigativo para o ensino de química. Monografia. Fortaleza, CE: Universidade Federal do Ceará.

Silva, W. B. D. 2017b. Análise de parâmetros relacionados a fraudes em leites UHT (Ultra High Temperatura) comercializados na cidade de Luz MG. Tese de doutorado. Luz, MG: (Faculdade de Filosofia, Ciências e Letras do Alto São Francisco.

Spink, J., \& Moyer, D. C. 2011. Definindo a ameaça à saúde pública por fraude alimentar. Journal of food Science, 76(9), R157-R163.

Tibola, C .S, Silva, S. A, Dossa A. A, \& Patrício, D.I. 2018. Fraude alimentar e adulteração economicamente motivadas no Brasil: incidentes e alternativas para minimizar a ocorrência. Journal of Food Science, 83, 2028-2038.

\section{Minicurrículo}

Sarah Ramila Batista de Oliveira. Graduanda do $8^{\circ}$ período do curso de Biomedicina pela Faculdade Única de Ipatinga (MG).

Lucas D’ Lúcio Sousa Moraes. Graduando do $8^{\circ}$ período do curso de Biomedicina pela Faculdade Única de Ipatinga (MG). Atualmente é estagiário da Vigilância Sanitária da Prefeitura de Coronel Fabriciano.

Cristina Pacheco Coelho. Possui graduação em Nutrição - Unileste (2008). Atualmente trabalha na Faculdade Única de Ipatinga/MG, onde coordena o curso de Nutrição. Especialista em Nutrição Clínica /GANEP/SP. Pós-Graduada em Nutrição Clínica e Estética /IPGS/RS. Pós-Graduada em Fitoterapia/Unileya/DF. Pós-Graduada em Nutrição Esportiva e Funcional /Instituto Valorize/ES. Graduada em Gastronomia - Unicesumar/ PR. Docente Faculdade Única dos cursos: Biomedicina, Enfermagem, Educação Física, Farmácia. Consultora em Unidades de Alimentação e Nutrição. Certificações Internacionais: University at Buffalo /USA e UCA/Argentina.

Como citar: Oliveira, S.R.B., Moraes, L.D.S., \& Coelho, C.P. 2021. Fraudes em alimentos industrializados. Pubsaúde, 5, $115 . \quad$ DOI: https://dx.doi.org/10.31533/pubsaude5.a115

Recebido: 12 dez. 2020.

Revisado e aceito: 5 jan. 2021.

Conflito de interesse: os autores declaram, em relação aos produtos e companhias descritos nesse artigo, não ter interesses associativos, comerciais, de propriedade ou financeiros que representem conflito de interesse.

Licenciamento: Este artigo é publicado na modalidade Acesso Aberto sob a licença Creative Commons Atribuição 4.0 (CC-BY 4.0). 